\title{
CIESIELSKI AND FRANKLIN SYSTEMS
}

\author{
GEGHAM G. GEVORKYAN \\ Department of Mathematics, Yerevan State University \\ Alex Manoukian St. 1, 375049 Yerevan, Armenia \\ E-mail:ggg@arminco.com
}

\begin{abstract}
A short survey of results on classical Franklin system, Ciesielski systems and general Franklin systems is given. The principal role of the investigations of Z. Ciesielski in the development of these three topics is presented. Recent results on general Franklin systems are discussed in more detail. Some open problems are posed.
\end{abstract}

The classical Franklin system, introduced by Ph. Franklin [11] in 1928, is a complete orthonormal system of continuous, piecewise linear functions with dyadic knots. In 1972 (see [6], [7]) Z. Ciesielski introduced the orthonormal spline systems of higher order that were later called Ciesielski systems. Z. Ciesielski and A. Kamont in [9] started the investigation of general Franklin systems with quasi-dyadic knots.

A detailed review of the studies on these systems up to the year 2000 is provided in the survey article by Z. Ciesielski and A. Kamont [10].

Let $\sigma_{N}=\left\{s_{n}: 0 \leq i \leq N\right\}$ be a partition of [0;1], admitting at most double knots, i.e.,

$$
0=s_{0}<s_{1} \leq \ldots \leq s_{N-1}<s_{N}=1
$$

and

$$
s_{i}<s_{i+2} \quad \text { for all } i, 0 \leq i \leq N-2 .
$$

Denote by $S(\sigma)$ the space of piecewise linear functions on [0;1] corresponding to the sequence of knots $\sigma$. By this we mean the space of functions linear on each interval $\left(s_{i} ; s_{i+1}\right)$, left-continuous at each $s_{i}$ (and right-continuous at $s_{0}$ ) and continuous at each $s_{i}, 1 \leq i \leq N-1$ satisfying $s_{i-1}<s_{i}<s_{i+1}$. The space $S(\sigma)$ is a linear space of dimension $N+1$.

Now, let $\sigma_{N}=\left\{s_{i}: 0 \leq i \leq N\right\}$ and $\sigma_{N+1}^{*}=\left\{s_{i}^{*}: 0 \leq i \leq N+1\right\}$ be a pair of partitions of $[0 ; 1]$ satisfying $(1),(2)$ such that $\sigma_{N+1}^{*}$ is obtained from $\sigma_{N}$ by adding one knot $s^{*}$. Note that $s^{*}$ may be different from all points of $\sigma_{N}$ (in this case we have

2000 Mathematics Subject Classification: 42C10, 46E15, 46E30, 42C25.

The paper is in final form and no version of it will be published elsewhere. 
$s^{*}=s_{i}^{*}$, for some $i$, and $s_{i-1}^{*}<s_{i}^{*}<s_{i+1}^{*}$ ), or $s^{*}=s_{i}$, for some $i$ (then $s_{i-1}^{*}<s_{i}^{*}=s^{*}=$ $\left.s_{i+1}^{*}<s_{i+2}^{*}\right)$. Now, there is a unique function $\varphi \in S\left(\sigma_{N+1}^{*}\right)$ that is orthogonal to $S\left(\sigma_{N}\right)$ with $\|\varphi\|_{2}=1$ and $\varphi\left(s^{*}\right)>0$. This function $\varphi$ is called the general Franklin function corresponding to the pair of partitions $\left(\sigma_{N} ; \sigma_{N+1}^{*}\right)$.

Definition 1 . Let $T=\left\{t_{n}: n \geq 0\right\}$ be a sequence of points from $[0 ; 1]$. The sequence $T$ is called admissible if $t_{0}=0, t_{1}=1, t_{n} \in(0 ; 1)$ for $n>1$, for each $t \in(0 ; 1)$ there are at most two different indices $n_{1}>n_{2}>1$ such that $t=t_{n_{1}}=t_{n_{2}}$, and $T$ is dense in $[0 ; 1]$.

For an admissible sequence $T=\left\{t_{n}: n \geq 0\right\}$ and $n>1$, let $\sigma_{n}=\left\{t_{n, i}: 0 \leq i \leq n\right\}$ be the partition of $[0 ; 1]$ obtained by the nondecreasing rearrangement of the first $n+1$ terms of the sequence $\left\{t_{n}: n \geq 0\right\}$, counting multiplicities. It is clear, that each $\sigma_{n}$ satisfies (1), (2) and $\sigma_{n}$ is obtained from $\sigma_{n-1}$ by adding one knot $t_{n}$.

Definition 2. Let $T$ be an admissible sequence of points. A general Franklin system corresponding to the sequence of knots $T$ is a sequence of functions $\left\{f_{n}: n \geq 0\right\}$ given by $f_{0}(t)=1, f_{1}=\sqrt{3}(2 t-1)$, and for $n \geq 2, f_{n}$ is the general Franklin function corresponding to the pair of partitions $\left(\sigma_{n-1} ; \sigma_{n}\right)$; i.e. $f_{n} \in S\left(\sigma_{n}\right), f_{n}$ is orthogonal to $S\left(\sigma_{n-1}\right),\left\|f_{n}\right\|_{n}=1$ and $f_{n}\left(t_{n}\right)>0$.

Definition 3. Let $T=\left\{t_{n}: n \geq 0\right\}$ be a sequence of points from [0;1] satisfying conditions;

1) $t_{0}=0, t_{1}=1,0<t_{2}<1$;

2) $t_{2^{k}+1}<t_{2^{k}+2}<\ldots<t_{2^{k+1}}, k=1,2, \ldots$;

3) between two neighbouring points from $\left\{t_{i}: 0 \leq i \leq 2^{k}\right\}$ there is a point in $\left\{t_{i}\right.$ : $\left.2^{k}+1 \leq i \leq 2^{k+1}\right\}$.

Then the sequence $T$ is called a quasi-dyadic partition of $[0 ; 1]$ and the corresponding general Franklin system is called a quasi-dyadic Franklin system.

The classical orthogonal Franklin system is obtained when the partition $T=\left\{t_{n}\right.$ : $n \geq 0\}$ is dyadic, i.e. $t_{0}=0, t_{1}=1$, and $t_{n}=\frac{2 m-1}{2^{k+1}}$ for $n=2^{k}+m, k=0,1, \ldots$, $1 \leq m \leq 2^{k}$. In $1928 \mathrm{Ph}$. Franklin [11] proved that this system is a basis in $C[0 ; 1$ ] (now called the classical Franklin system). It is the first example of orthogonal basis in $C[0 ; 1]$. Before Ph. Franklin in 1910, A. Haar [19] introduced an orthogonal complete system with the property: its Fourier expansion of any continuous function converges uniformly. But the functions of the Haar system are not continuous, consequently the Haar system is not a basis in $C[0 ; 1]$.

Afterwards the Franklin system was not studied until the work of Z. Ciesielski, who started the systematic investigation of this system. In 1966 Z. Ciesielski [5] proved the exponential estimates for functions of the classical Franklin system.

Theorem 1 (Z. Ciesielski). Let $\left\{f_{n}: n \geq 0\right\}$ be the classical Franklin system. Then there exist constants $C>0$ and $q \in(0 ; 1)$ such that for each $n=2^{k}+m, k=0,1,2, \ldots$, $1 \leq m \leq 2^{k}$

$$
\left|f_{n}(x)\right| \leq C \sqrt{n} q^{n\left|x-t_{n}\right|}
$$

where $t_{n}=\frac{2 m-1}{2^{k+1}}$. 
The estimate (3) plays an important role in the investigation of the classical Franklin system. Here we mention some of the principal results.

Let $M(f, x)$ be the Hardy-Littlewood maximal function of an integrable function $f$ and let $S_{N}(f, x)$ be the partial sum of $f$ with respect to the classical Franklin system. Moreover let

$$
S^{*}(f, x)=\sup _{1 \leq N<\infty}\left|S_{N}(f, x)\right|
$$

be the majorant of the Fourier-Franklin series.

Theorem 2 (Z. Ciesielski [5]). For any $f \in L^{1}[0 ; 1]$

$$
S^{*}(f, x) \leq C \cdot M(f, x),
$$

where $C$ is an absolute constant.

Later Z. Ciesielski and A. Kamont [9] proved that Theorem 2 is true for any general Franklin system.

Theorem 3 (S. V. Bochkarev [1]). The classical Franklin system is an unconditional basis in all reflexive Orlicz spaces; in particular, in the spaces $L^{p}$ for $1<p<\infty$.

TheOREM 4 (P. Wojtaszczyk [24]). The classical Franklin system is an unconditional basis in $H^{1}[0 ; 1]$.

Applying the classical Franklin system, S. V. Bochkarev [3] constructed a basis in the space $A$ of complex-valued functions analytic inside the unit disk and continuous in the closed unit disk. For the classical Franklin system $\left\{f_{n}: n \geq 0\right\}$ S. V. Bochkarev defined

$$
F_{n}(x)= \begin{cases}f_{n}\left(\frac{x}{\pi}\right), & \text { for } x \in[0 ; \pi], \\ f_{n}\left(-\frac{x}{\pi}\right), & \text { for } x \in[-\pi ; 0],\end{cases}
$$

and

$$
G_{0}(x)=\frac{1+i}{2 \sqrt{\pi}}, \quad G_{n}(x)=\frac{1}{\sqrt{2 \pi}}\left[F_{n}(x)+i \widetilde{F}_{n}(x)\right]
$$

where the function

$$
\widetilde{F}_{n}(x)=-\lim _{\varepsilon \rightarrow+0} \frac{1}{\pi} \int_{\varepsilon}^{\pi} \frac{F_{n}(x+t)-F_{n}(x-t)}{2 \tan \frac{t}{2}} d t
$$

is the trigonometric conjugate of $F_{n}(x)$

Theorem 5 (S. V. Bochkarev). The system of functions

$$
G_{n}(z) \equiv G_{n}\left(r e^{i x}\right)=\frac{1}{2 \pi} \int_{-\pi}^{\pi} G_{n}(t) P(r, x-t) d t,
$$

where $P(r, x)$ is the Poisson kernel, forms a basis in the space $A$.

Combining the results from [5], [1], [14], [23] we can formulate

THEOREM 6. Let $\left\{f_{n}: n \geq 0\right\}$ be the classical Franklin system and $p \in(0 ;+\infty)$. Then the following conditions are equivalent:

1) $\sum_{n=1}^{\infty} a_{n} f_{n}(x)$ converges unconditionally in $L^{p}$;

2) $\left\{\sum_{n=0}^{\infty} a_{n}^{2} f_{n}^{2}(x)\right\}^{1 / 2} \in L^{p}$;

3) $\sup _{N}\left|\sum_{n=0}^{N} a_{n} f_{n}(x)\right| \in L^{p}$. 
Theorem 7 (G. G. Gevorkian [13]). Let E be a given Lebesgue measurable subset of $[0 ; 1]$ of positive measure and let $\left\{f_{n}: n \geq 0\right\}$ be the classical Franklin system. Then the following conditions are equivalent:

1) $\sum_{n=1}^{\infty} a_{n} f_{n}(x)$ converges a.e. on $E$;

2) $\sum_{n=0}^{\infty} a_{n}^{2} f_{n}^{2}(x)<\infty$ a.e. on $E$;

3) $\sup _{N}\left|\sum_{n=0}^{N} a_{n} f_{n}(x)\right|<\infty$ a.e. on $E$;

4) $\sum_{n=1}^{\infty} a_{n} f_{n}(x)$ converges unconditionally in measure on $E$.

In my opinion the main open problems for classical Franklin series are related to Cantor's type uniqueness theorem.

Problem 1. Let $\left\{f_{n}: n \geq 0\right\}$ be the classical Franklin system and let $\sum_{n=0}^{\infty} a_{n} f_{n}(x)=0$ for each $x \in[0 ; 1]$. Does it follow that $a_{n}=0$ for all $n=0,1,2, \ldots$ ?

It is well-known [4], [5] that $\left|\sum_{n=0}^{N} f_{n}(0) f_{n}(t)\right|<C \cdot N \cdot q^{N t}$, where $C, q$ are some absolute constants and $0<q<1$. Hence, there is a series $\sum_{n=0}^{\infty} a_{n} f_{n}(t)$ convergent to zero for $t \in(0 ; 1]$ with $a_{0} \neq 0$.

Note, that $\left|f_{n}(0)\right|<C \sqrt{n}$ and $\limsup _{n \rightarrow 0} \frac{\left|f_{n}(0)\right|}{\sqrt{n}}>0$ (see [4], [5]).

Problem 2. Let $\left\{f_{n}: n \geq 0\right\}$ be the classical Franklin system. Do the conditions $\sum_{n=0}^{\infty} a_{n} f_{n}(x)=0$ for $x \in(0 ; 1]$ and $a_{n}=o(\sqrt{n})$ imply that $a_{n}=0$ for all $n=0,1,2, \ldots$ ?

Problem 3. Let $\left\{f_{n}: n \geq 0\right\}$ be the classical Franklin system and let $E$ be some finite or countable set. Do the conditions $\sum_{n=0}^{\infty} a_{n} f_{n}(x)=0$ for $t \in[0 ; 1] \backslash E$ and $a_{n}=o(\sqrt{n})$ imply that $a_{n}=0$ for all $n=0,1,2, \ldots$ ?

The uniqueness theorem of another type was proved in [12], where we found the following necessary and sufficient conditions for the Franklin series to be a Fourier-Franklin series.

Theorem 8 (G. G. Gevorkian [12]). The classical Franklin series $\sum_{n=0}^{\infty} a_{n} f_{n}(x)$ is a Fourier-Franklin series of a function $f \in L^{1}[0 ; 1]$ if and only if it converges a.e. to $f$ and

$$
\liminf _{\lambda \rightarrow+\infty} \lambda \cdot\left|\left\{x \in[0 ; 1]: \sup _{N}\left|\sum_{n=0}^{N} a_{n} f_{n}(x)\right|>\lambda\right\}\right|=0 .
$$

In particular, if $f=0$, then $a_{n}=0$ for all $n \geq 0$.

In $[15,16,17]$ several types of regularities of partitions were defined.

Let $T=\left\{t_{i}: i \geq 0\right\}$ be an admissible sequence of points and let $\sigma_{n}=\left\{0=t_{n, 0}<\right.$ $\left.t_{n, 1} \leq \ldots \leq t_{n, n-1}<t_{n, n}=1\right\}$ be a partition of $[0 ; 1]$ obtained by nondecreasing rearrangement of $T_{n}=\left\{t_{i}: 0 \leq i \leq n\right\}$. Denote $I_{n, i}=\left[t_{n, i-1}: t_{n, i}\right]$ and $\lambda_{n, i}=\left|I_{n, i}\right|=$ $t_{n, i}-t_{n, i-1}$.

Definition 4. Let $T$ be an admissible sequence of points. We say that $T$ satisfies the strong regularity condition with parameter $\gamma \geq 1$ if for $n \geq 1$ and $2 \leq i \leq n$

$$
\frac{1}{\gamma} \lambda_{n, i-1} \leq \lambda_{n, i} \leq \gamma \lambda_{n, i-1}
$$


Definition 5. Let $T$ be an admissible sequence of points. We say that $T$ satisfies the strong regularity condition for pairs with parameter $\gamma \geq 1$ if for $n \geq 1$ and $2 \leq i \leq n$

$$
\frac{1}{\gamma}\left(\lambda_{n, i-1}+\lambda_{n, i}\right) \leq \lambda_{n, i}+\lambda_{n, i+1} \leq \gamma\left(\lambda_{n, i-1}+\lambda_{n, i}\right)
$$

with the convention $\lambda_{n, 0}=\lambda_{n, n+1}=0$.

M. P. Poghosyan [20, 21] proved the analogues of theorems 7 and 8 for quasi-dyadic Franklin systems generated by a partition $T$ satisfying the strong regularity condition.

Problem 4. Are the analogues of theorems 7 and 8 true for an arbitrary general Franklin system?

The problem of unconditionality of general Franklin systems was treated in [15] and [18], where some partial results were obtained, under additional conditions on the structure and regularity of the sequence of knots. Developing the method of [18], we proved that for any admissible sequence of knots $T$, the corresponding Franklin system is an unconditional basis in $L^{p}[0 ; 1], 1<p<\infty$. Moreover, we show that each general Franklin system normalized in $L^{p}[0 ; 1], 1<p<\infty$, is a greedy basis in this space.

Theorem 9 (G. G. Gevorkyan, A. Kamont [16]). Let $T=\left\{t_{n}: n \geq 0\right\}$ be an admissible sequence of knots. Then the corresponding general Franklin system is an unconditional basis in each $L^{p}[0 ; 1], 1<p<\infty$.

Theorem 10 (G. G. Gevorkyan, A. Kamont [16]). Let $T=\left\{t_{n}: n \geq 0\right\}$ be an admissible sequence of knots with the corresponding general Franklin system $\left\{f_{n}: n \geq 0\right\}$. For given $p, 1<p<\infty$, let $f_{n, p}=f_{n} /\left\|f_{n}\right\|_{p}$. Then $\left\{f_{n, p}: n \geq 0\right\}$ is a greedy basis in $L^{p}[0 ; 1]$.

In [15] a general Franklin system was discussed as a basis in $H^{1}[0 ; 1]$, but only for quasi-dyadic partitions of $[0 ; 1]$. Theorem 5.3 from [15] states that for any quasi-dyadic sequence of partitions, the general Franklin system is a basis in $H^{1}[0 ; 1]$ iff it is an unconditional basis in $H^{1}[0 ; 1]$, and both these conditions are equivalent to the strong regularity of the corresponding sequence of partitions. It turns out that for general Franklin systems corresponding to partition without any structural constraints, the property of being a basis in $H^{1}[0 ; 1]$ and being an unconditional basis in $H^{1}[0 ; 1]$ are no longer equivalent. Clearly, for quasi-dyadic sequences of partitions strong regularity and strong regularity for pairs are equivalent.

Theorem 11 (G. G. Gevorkyan, A. Kamont [17]). Let $T_{n}=\left\{t_{n}: n \geq 0\right\}$ be an admissible sequence of knots with the corresponding general Franklin system $\left\{f_{n}: n \geq 0\right\}$. Then $\left\{f_{n}: n \geq 0\right\}$ is a basis in $H^{1}[0 ; 1]$ if and only if $T$ satisfies the strong regularity condition for pairs with some parameter $\gamma>1$.

Theorem 12 (G. G. Gevorkyan, A. Kamont [17]). Let $T_{n}=\left\{t_{n}: n \geq 0\right\}$ be an admissible sequence of knots with the corresponding general Franklin system $\left\{f_{n}: n \geq 0\right\}$. Then $\left\{f_{n}: n \geq 0\right\}$ is an unconditional basis in $H^{1}[0 ; 1]$ if and only if $T$ satisfies the strong regularity condition with some parameter $\gamma>1$.

One of the main new ideas in the proofs of theorems 9-12 is a new choice of "canonical" intervals associated with a general Franklin function. In [15], [18] the function $f_{n}$ with 
$n=2^{j}+k$ has been associated with the interval $\{n\}=\Delta_{j, k}$, into which the point $t_{n}$ is inserted, and all estimates, splittings and reorderings for a general Franklin system have been done with respect to positions of $\{n\}$ or $t_{n}$.

Now we describe the new choice of the canonical intervals (intervals $J$ ). Let

$$
\sigma_{n}=\left\{0=t_{n, 0}<t_{n, 1} \leq \ldots \leq t_{n, n-1}<t_{n, n}=1\right\}
$$

be a partition of $[0 ; 1]$ obtained by nondecreasing rearrangement of $T_{n}=\left\{t_{i}: 0 \leq i \leq n\right\}$ and $t_{n}=t_{n, k}$ for some $k, 1 \leq k \leq n-1$. Consider the following intervals:

$$
I=\left[t_{n, k-1} ; t_{n, k+1}\right], \quad I^{-}=\left[t_{n, k-2} ; t_{n, k}\right], \quad I^{+}=\left[t_{n, k} ; t_{n, k+2}\right]
$$

and denote

$$
\nu=|I|, \quad \nu^{-}=\left|I^{-}\right|, \quad \nu^{+}=\left|I^{+}\right|, \quad \mu=\min \left\{\nu^{-}, \nu, \nu^{+}\right\} .
$$

Now, let $I^{*}=\left[t_{n, i} ; t_{n, i+2}\right]$ be one of the intervals $I^{-}, I, I^{-}$such that $\mu=\left|I^{*}\right|$, and consider its left and right parts $I^{i, l}=\left[t_{n, i} ; t_{n, i+1}\right], I^{i, r}=\left[t_{n, i+1} ; t_{n, i+2}\right]$. Finally, let $J_{n}$ be one of the intervals $I^{i, l}, I^{i, r}$ such that $\left|J_{n}\right|=\max \left\{\left|I^{i, l}\right|,\left|I^{i, r}\right|\right\}$. For canonical intervals we have the following

Proposition. Let $T=\left\{t_{n}: n \geq 0\right\}$ be an admissible sequence of points with the corresponding Franklin system $\left\{f_{n}: n \geq 0\right\}$. Let $k, l \geq 0$ be such that $t_{k} \leq t_{l}$ and there is no integer $i \leq \max \{k, l\}$ with $t_{i} \in\left(t_{k} ; t_{l}\right)$. Then

1) $\operatorname{card}\left\{n: J_{n}=\left[t_{k} ; t_{l}\right]\right\} \leq 5$;

2) $\operatorname{card}\left\{n: J_{n} \subset\left[t_{k} ; t_{l}\right]\right.$ and $\left.\left|J_{n}\right|>\left|\left[t_{k} ; t_{l}\right]\right| / 2\right\} \leq 25$.

Problem 5. Let $T=\left\{t_{n}: n \geq 0\right\}$ be a sequence of simple knots, $i$. e. $t_{i} \neq t_{j}$ for $i \neq j$, and $\left\{f_{n}: n \geq 0\right\}$ be the corresponding general Franklin system.

Is the system of functions $G_{n}(z)$, defined by (4)-(7), a basis in the space $A$ ?

PROBlem 6. If the answer of problem 5 is negative, then describe sequences $T$ for which the functions $G_{n}(z)$ form a basis in the space $A$.

Many properties of the classical Franklin system are fulfilled for Ciesielski systems with dyadic knots. But some of them are not known. In particular, the equivalence of Paley's function and majorant of partial sums for Ciesielski systems is checked only for $p \geq 1$.

Problem 7. Let $\left\{F_{n}^{m}(x)\right\}$ be the Ciesielski system of order $m>2$. Are the following conditions equivalent for $0 \leq p<1$ ?

1) The series $\sum_{n=1}^{\infty} a_{n} F_{n}^{m}(x)$ converges unconditionally in $L^{p}$;

2) $\left\{\sum_{n=0}^{\infty}\left(a_{n} F_{n}^{m}(x)\right)^{2}\right\}^{\frac{1}{2}} \in L^{p}$;

3) $\sup _{N}\left|\sum_{n=0}^{N} a_{n} F_{n}^{m}(x)\right| \in L^{p}$.

For the orthonormal spline system with dyadic knots on $R$ (Strömberg's wavelet system) the answer is positive (Z. Ciesielski, G. G. Gevorkyan [8]).

Recently, A. Shadrin [22] solved in the positive de Boor's conjecture on the uniform boundedness of $L^{\infty}$-norms of the orthogonal projections onto the space of splines of any (fixed) order with arbitrary knots. This implies that for any partition with simple knots the corresponding general Ciesielski system is a basis in $C(0 ; 1)$. 
Problem 8. Does the general Ciesielski system form an unconditional basis in $L^{p}[0 ; 1]$ for $1<p<\infty$ ?

Now we consider periodic Franklin systems with simple knots.

Let $T=\left\{t_{n}: n \geq 1\right\}$ be a sequence of points from $[0 ; 1]$ and $t_{i} \neq t_{j}$ for $i \neq j$. Denote by $S_{n}(T)$ the space of piecewise linear and continuous functions on [0;1] with knots $t_{1}, t_{2}, \ldots, t_{n}$ and periodic with period 1 .

Definition 6. A general periodic Franklin system corresponding to the sequence of knots $T$ is a sequence of functions $\left\{f_{n}: n \geq 1\right\}$ given by $f_{1}(t)=1$, and for $n \geq 2, f_{n} \in S_{n}(T)$, $f_{n} \perp S_{n-1}(T),\left\|f_{n}\right\|_{2}=1$ and $f_{n}\left(t_{n}\right)>0$.

Problem 9. Is the periodic general Franklin system unconditional basis in the space $L^{p}[0 ; 1], 1<p<\infty$ ?

Problem 10. Let $\left\{f_{n}: n \geq 1\right\}$ be periodic general Franklin system corresponding to the sequence of knots $T$. Find necessary and sufficient conditions on the sequence $T=\left\{t_{n}\right.$ : $n \geq 1\}$ for the system $\left\{f_{n}: n \geq 1\right\}$ to be a basis or an unconditional basis in $\operatorname{ReH}^{1}$.

In $1985 \mathrm{~S}$. V. Bochkarev [2] proved that if $\left\{f_{n}: n \geq 1\right\}$ is the periodic classical Franklin system on $[0 ; 2 \pi]$, then the system of functions $\frac{1}{2 \pi}, \widetilde{f}_{2}, \widetilde{f}_{3}, \ldots$ form a basis in the space of continuous and $2 \pi$-periodic functions, where $\widetilde{g}$ is the trigonometric conjugate of $g$.

Problem 11. Let $\left\{f_{n}: n \geq 1\right\}$ be periodic general Franklin system on $[0 ; 2 \pi]$. Is the system of functions $\frac{1}{2 \pi}, \widetilde{f}_{2}, \widetilde{f}_{3}, \ldots$ a basis in the space of continuous and $2 \pi$-periodic functions?

Problem 12. If the answer to problem 11 is negative, then what is the necessary and sufficient condition on the sequence $T=\left\{t_{n}: n \geq 1\right\}$ under which the corresponding system $\frac{1}{2 \pi}, \widetilde{f}_{2}, \widetilde{f}_{3}, \ldots$ is a basis in the space of continuous and $2 \pi$-periodic functions?

Added in proof. After this paper was submitted for publication, some of the problems listed above have been solved. Problem 9 has been solved in the positive in K. A. Keryan, Unconditional basis property of the periodic general Franklin system in $L_{p}[0,1], 1<p<\infty$, to appear in Izv. Nats. Akad. Nauk Armenii, Mat. (in Russian), English transl. in J. Contemp. Math. Anal. Problem 10 has been solved in K. A. Keryan and M. P. Poghosyan, Periodic Franklin systems as bases in $H^{1}[0,1]$, to appear in Izv. Nats. Akad. Nauk Armenii, Mat. (in Russian), English transl. in J. Contemp. Math. Anal.; the result is a periodic version of Theorems 11 and 12. Problems 5 and 11 have been answered in the negative, and some partial answers to Problems 6 and 12 have been found. The summary of these results is contained in G. G. Gevorkyan and A. Kamont, Some properties of the conjugate general Franklin system (in Russian), to appear in Dokl. Nats. Akad. Nauk Armenii, and the full version with proofs is in preparation for publication.

\section{References}

[1] S. V. Bochkarev, Some inequalities for the Franklin series, Analysis Mathematica 1 (1975), 249-257. 
[2] S. V. Bochkarev, The conjugate Franklin system is a basis in the space of continuous functions, Dokl. AN SSSR 285 (1985) 521-526 (in Russian).

[3] S. V. Bochkarev, The existence of a basis in the space of functins analytic in the disk, and some properties of the Franklin system, Mat. Sb. 95 (137) (1974), 3-18 (in Russian).

[4] Z. Ciesielski, Properties of the orthonormal Franklin system, Studia Math. 23 (1963) 141157.

[5] Z. Ciesielski, Properties of the orthonormal Franklin system II, Studia Math. 27 (1966), 289-323.

[6] Z. Ciesielski, Constructive function theory, Studia Math. 53 (1975), 277-301.

[7] Z. Ciesielski, Spline bases in funcion spaces, in: Proc. of the Conference on Approximation Theory, Poznań, 1972, 49-54.

[8] Z. Ciesielski and G. G. Gevorkyan, Franklin spline orthogonal system in $L^{p}(R)$ with $0<$ $p \leq 1$, in: Acta Sci. Math. (Szeged) 66 (2000), 211-226.

[9] Z. Ciesielski and A. Kamont, Projections onto piecewise linear functions, Functiones et Approximatio 29 (1997), 129-143.

[10] Z. Ciesielski and A. Kamont, Survey on the orthonormal Franklin system, in: Approximation Theory, Darba, Sofia, 2002, 84-132.

[11] Ph. Franklin, A set of continuous orthogonal functions, Math. Annalen 100 (1928), 522529 .

[12] G. G. Gevorkyan, On uniqueness of Franklin series, Mat. Zametki 24 (1989), 51-58 (in Russian).

[13] G. G. Gevorkyan, On series in the Franklin system, Analysis Mathematica 3 (1989), 87-114 (in Russian).

[14] G. G. Gevorkyan, Some theorems on unconditional convergence and a majorant of Franklin series and their application to the spaces $\operatorname{Re}\left(H^{p}\right)$, Proceedings of the Steklov Institute of Mathematics (1992), 49-76.

[15] G. G. Gevorkyan and A. Kamont, On general Franklin systems, Dissertationes Mathematicae (Rozprawy Matematyczne) 374 (1998).

[16] G. G. Gevorkyan and A. Kamont, Unconditionality of general Franklin systems in $L^{p}[0 ; 1]$, $1<p<\infty$, Studia Math. 164 (2004), 161-201.

[17] G. G. Gevorkyan and A. Kamont, General Franklin systems as bases in $H^{1}$, Studia Math. 167 (2005), 259-292.

[18] G. G. Gevorkyan and A. A. Sahakian, Unconditional basis property of general Franklin systems, J. Contemp. Math. Anal. 20 (2000), 2-22.

[19] A. Haar, Zur Theorie der orthogonalen Functionensysteme, Math. Ann. 10 (1910), 331371.

[20] M. P. Poghosyan, Uniqueness of series by general Franklin system, J. Contemp. Math. Anal. 20 (2000), 75-81.

[21] M. P. Poghosyan, Almost everywhere convergence of series by general Franklin system, J. Contemp. Math. Anal. 20 (2000), 61-73.

[22] A. Yu. Shadrin, The $L^{\infty}$-norm of the $L_{2}$-spline-projector is bounded independently of the knot sequence: A proof of de Boor's conjecture, Acta Mathematica 187 (2001), 59-137.

[23] P. Sjölin and J. O. Strömberg, Basis properties of Hardy spaces, Ark. Mat. 21 (1983), 111-125.

[24] P. Wojtaszczyk, The Franklin system is an unconditional basis in $H^{1}$, Ark. Mat. 20 (1982), 293-300. 